\title{
LIGHT TRESPASS COUNTERMEASURES
}

\author{
DR. D. A. SCHREUDER \\ Institute for Road Safety Research SWOV, The Netherlands
}

\section{INTRODUCTION}

Human life is geared to the rhythm of day and night. The day is committed to work and activity, and the night to rest, to leisure, but also to contemplation on the universe. Stars are the link between the earth-bound human existence and the eternal and infinite cosmos. So, humanity needs the night, requires the darkness - even if darkness is frightening and is considered as the personification of danger and evil.

Since just over one century, this rhythm is broken. Industrialization changed society, and provided the technology to implement these changes. Our modern society is a very complicated system. Production and consumption usually are far apart. Production and consumption include information and means for cultural or physical recreation and enjoyment. This requires a very large amount of transportation; a large proportion of this is road transportation of persons or goods. Parts of it have to be made at night in the absence of (natural) daylight; life must also go on after sunset! Nighttime transport requires outdoor lighting. Outdoor lighting, however, has serious drawbacks. It causes disturbance and discomfort for people who have nothing to do with the transportation for which the lighting is installed. Here we are facing "light trespass." Light trespass has three major groups of victims: the astronomers, the residents and the naturalists.

\section{LIGHT TRESPASS}

Light trespass is a matter of major concern for the astronomers. Usually it presents itself in the form of a sky glow in the vicinity of large urban or industrial concentrations. The luminance of sky glow can be expressed by "Walker's Law" which is written as: $\log p=-4.7 \log R-\log L$ (after Anon, 1984; see Walker 1973) in which $p$ is the ratio between the observed sky glow as measured in the direction of the source under an elevation of $45^{\circ}$ and the natural background radiation, $R$ the distance to the source (in $\mathrm{km}$ ), and $\mathrm{L}$ the total luminous flux of the outdoor lighting in the source (in lumen). The background radiation is usually taken as amounting to $2.10^{-4} \mathrm{~cd} / \mathrm{m}^{2}$. Furthermore, there is a relation between the population of the city and its total outdoor luminous flux. A rule of thumb suggests one thousand lumens per capita. "Walker's Law" is established for cities in the southwestern United States. It seems to be applicable for other locations as well. Further research is urgently required. 
The sky glow is a result of all outdoor lighting installations. In the urban areas that are prevalent in the southwestern U.S., the contribution of road lighting seems to be small compared to the contribution of other outdoor lighting, particularly billboard and advertisement lighting. According to Finch et al. (1979) for the city of San Carlos, CA, the street lighting contributes only $14 \%$ to the total (direct and reflected) upward luminous flux, and $86 \%$ comes from other sources. It is not known how these ratios are for other parts of the world where rather different practices of outdoor lighting may be found. Particularly it would be of interest to know how far Walker's Law applies for continental Europe, and what would be the portion of street lighting in the upward luminous flux, as the street lighting is mostly by semi cut-off lanterns and billboard lighting is used hardly at all.

It should be kept in mind that an important part of astronomical observations are made in the infrared and ultraviolet regions, and another large part of the observations involve spectroscopy. This implies that it is not enough to consider the "white" visible light of the sky glow; its spectral composition is also important.

This paper deals primarily with road lighting as a source of light trespass from which astronomers may suffer. First, we will briefly discuss the function of road lighting, concentrating on its contribution to the prevention of nighttime traffic accidents. The next point will be the measures that can be taken to prevent or at least reduce the light trespass. Finally, we will say a few words on what astronomers and lighting engineers can do (see also Schreuder, 1987).

\section{THE FUNCTION OF ROAD LIGHTING}

The primary function of transport lighting is to permit societal activities during the time that daylight is lacking. The two major categories of transport lighting are lights carried by traffic participants (vehicles) themselves and the public (overhead, street) lighting.

The function of road lighting is derived from the visual task aspects of the traffic participants. Here we will concentrate on (car) drivers, as their task is the most demanding. The task of drivers is twofold: to reach the destination of the trip, and to avoid collisions while doing so. Broadly speaking, the first task involves the selection of the route and maintaining it; the second is related to the avoidance of incidents and obstacles. Both task aspects involve the movements of the vehicle and changes therein, longitudinal and lateral position on the road, and the time derivatives of these (speed, decelerations, accelerations). It should be stressed that taking part in traffic, particularly as the driver of a car, is primarily a decision-making task, and not in the first place a visual task, as is often stated. Details of the "model" for traffic participation are given by Schreuder (1984).

The decisions are made primarily on the basis of visual information. Road lighting is essential to provide the information also in the dark. The two task aspects require specific information (the demand); the lighting installation provides the information (the supply). In order to permit adequate road transport, the supply should exceed or at least equal the demand (see Schreuder, 1977). The demands do not relate exclusively to motor traffic. They relate as well to the security and the amenity of residents. One may look into the accident statistics to find out whether the requirements regarding the safety goals are met. 
This has been done on a large scale (OECD, 1971; CIE, 1988; Fisher and Hall, 1978; Schreuder, 1983) resulting in the statement that "good" road lighting as compared with "poor" lighting may result in a reduction of about $30 \%$ in nighttime injury accidents for major urban roads and for rural motorways.

For motor traffic the quality of the lighting is primarily determined by the road surface luminance (the "brightness") and not the illuminance (the amount of light falling on the surface). Usually, one considers a value of $1 \mathrm{~cd} / \mathrm{m}^{2}$ or even more as the minimum luminance for important traffic thoroughfares both in urban and rural areas. For less important roads, luminance levels of $0.5 \mathrm{~cd} / \mathrm{m}^{2}$ or lower are recommended. These recommendations of the International Lighting Commission are widely accepted and used in most countries of the world (CIE, 1977a). However, further research is urgently needed in this matter, as the luminance values quoted above are, in fact, based mainly on practical experience.

\section{TECHNICAL ASPECTS OF ROAD LIGHTING}

Cost factors have always played a major role in road lighting policy. The costs of installing and maintaining the lighting are more or less fixed for an installation of a certain type. The lamp costs and the energy costs, however, may vary considerably from one type of lamp to another and from one location to another as well! Consequently, it is not possible to indicate one specific type of lamp that is most economic. In fact, there are several families of lamps that are widely used for road lighting: incandescent lamps; low-pressure sodium-vapor discharge lamps; high-pressure sodium-vapor discharge lamps. Each of these families consists of many types that are similar in construction but different in lumen output and consequently in dissipated wattage.

The luminous efficacy of the different lamp families and types is widely different. The incandescent lamps have an efficacy (expressed in lumen per watt) of some 7 to 15 ; fluorescent tubes 45 to 95 ; high-pressure mercury 40 to 100 ; lowpressure sodium 140 to over 200; and high-pressure sodium 90 to 140 . Because, however, some lamps are cheap and others expensive, each lamp type will have its own field of application. Incandescent lamps are not used any longer in modern installations for "normal" roads; they are widely used for floodlighting and for decorative lighting in ancient city centers, etc.

Another important difference is the size of the lamps. This is obviously important for the size of the lanterns that must be able to accommodate them, but also for the dimensions of the optics.

The color characteristics of the lamps are important. Incandescent lamps emit a continuous spectrum similar to the black-body radiation. As a result of the permissible temperature of the incandescent filament, most energy is emitted in the infrared region and in the adjoining red. Thus, the low efficacy and the yellowish color of these lamps. Low-pressure vapor-discharge lamps emit essentially line spectra only. The lines of mercury are in the blue and green but particularly in the (invisible) ultraviolet. In order to increase the amount of visible light and thus the efficacy, fluorescent material is added that transforms the (monochromatic) ultraviolet light in (heterochromatic) visible light. The light of fluorescent tubes is therefore not monochromatic any longer. Its actual color can be selected in a wide range by applying appropriate fluorescent materials (the so-called phosphors). The emission of low-pressure sodium-vapor discharges is 
monochromatic as well, the wavelength being about $590 \mathrm{~nm}$. Because this is in the middle of the visible part of the electromagnetic spectrum, the discharge is used directly. The result is a monochromatic light source with very high efficacy, but which is not capable of rendering any colors other than yellow (and black). For transport lighting the monochromatic light is not considered as a drawback; for the reduction in the negative aspects of light trespass it is a great advantage. In fact, the application of low-pressure sodium lamps is considered as the most important single remedy for the interference with astronomical observation (Anon, 1985, 1985a; Crawford, 1983, 1985; IAU/CIE, 1980). Electric discharges in vapor under high pressure are not monochromatic as a result of the widening of the spectral lines. The emission spectrum is not fully continuous; to a certain extent the original lines can be recognized in the spectrum of the lamp. The highpressure sodium lamps show the original but widened sodium lines. Other elements are sometimes added to improve color rendering, thereby reducing, however, the efficacy. In high pressure discharge lamps one must take into account the additional emission of the discharge tube; that usually will be extremely hot and will act as an incandescent body. The fluorescent bulb of high pressure mercury lamps is relatively large in respect to the discharge tube. Consequently, the optical system of the lantern must be designed in relation to this.

Lamps emit light in nearly all directions. A lighting scheme, however, requires usually a strict light control. One must ensure that the light is directed towards the objects to be lighted, and that no light shines in other directions particularly in directions where it might cause glare or light trespass.

One may discern two ways of light control: simple screening and actually directing light in the desired directions. The first is simply a matter of shields and baffles. Directing the light in the desired directions requires the application of optical means: mirrors, lenses or prisms. Basically, the optical control is realized by constructing a set of optical images of the light source (often but not always the discharge tube of the lamp) into the object to be illuminated. In the case of road lighting, this means to construct a number of optical images of the light source on the road surface in such a way that, taking into account the way the surface reflects the light, the desired distribution of the luminance of the road surface (as it presents itself to an observer in a specified location) is realized. In the case of lighting of sports facilities, the same procedure is followed aimed at a specific distribution of the illuminance on the field.

The accuracy in which the light control can be realized depends on the divergence of the optical images. Here two factors have to be mentioned. The light sources are not very small relative to the focal length. This leads to a considerable beam divergence (or beam aperture). Furthermore, the optical systems are of a simple construction. Lenses are usually made of pressed glass or extruded plastic elements, and mirrors usually are formed of pressed aluminum. Considerable errors - in an optical sense - are unavoidable. This is added to the scatter of light from dust, grime, water, etc., on the exterior surfaces of the lanterns. One might add here that the latter are reduced considerably when closed lanterns are used. In the case of interference with astronomical observations, it is primarily the light above the horizon that matters; that light may be reduced by applying lanterns that have a flat (transparent) lower surface, a "flat bowl," so to speak. In this case, no light at all can be directed over the horizon. 
The road surface is the third element of the lighting scheme because it is supposed to reflect the light. In many cases the amount of reflected light - and consequently the luminance - depends on the direction of light incidence and of observation. The "reflection factor" actually is a tensor. Road surfaces show this effect to a high degree as a result of the glancing observation angles that are inherent to motorized road traffic, particularly when roads are damp or wet.

In designing effective road lighting installations, this effect is used to a considerable degree. The lanterns are designed so that most of the light is emitted in directions close to, and lower than, the horizon. In this way, the highest use is made from the specular characteristics of the road. Lanterns showing such emission (or luminous intensity distributions) are widely used in continental Europe, particularly in roads with a traffic function. There is, however, an optimum. When the light is emitted by the lanterns in an almost horizontal direction, as is often done in countries outside Europe, the resulting road surface luminance is lower.

Both practical experience with modern, skid-proof road surfaces and calculations prove conclusively that there really is no ground at all to use "non-cut-off" lanterns in road lighting for traffic routes. For residential areas, shopping areas and pedestrian precincts, one prefers a lively surrounding that is focused on the needs of pedestrians. Non-cut-off lanterns may contribute to such lively surroundings. However, such non-cut-off lanterns may contribute to light trespass.

As regards the road surface, reducing the reflection factor (i.e. selecting a darker surface) does not reduce the light spill; contrary to this, the light spill will increase because a larger lamp (a higher lumen output) must be used in order to arrive at the same road surface luminance.

\section{REMEDIAL MEASURES}

The considerations given in the foregoing sections point towards several possibilities to avoid or at least to reduce the disturbance for astronomical observations caused by light trespass:

a) Reduce the total amount of light: This obviously is the final solution as regards the astronomical observations. However, it cannot be done in full as other equally important aspects of our social life require nighttime activities and consequently artificial light at night. Nevertheless, a fair number of light sources can be shut off without any damage. Furthermore, in many cases, light sources can be replaced by smaller sources emitting less light (other, even more effective possibilities are discussed later). An important way to improve the situation is to guarantee that most lights, particularly those that contribute most to light pollution, like sports stadium lighting, are not used after a certain time. Many local bylaws and ordinances impose time restrictions in the use of such lighting installations.

b) Reduce the spectral spread of the light emission: An effective countermeasure against the interference of light trespass is the reduction of the width of the spectral range of the emitted light. The most effective way available at present is the use of (quasi) monochromatic light sources, particularly the use of low-pressure sodium-vapor lamps. These lamps emit a very narrow spectral band in the yellow part of the spectrum. Two advantages are obvious: Other 
spectral regions are not involved, so that observations in other spectral regions are hardly affected. Secondly, as the yellow line is close to the maximum of the sensitivity of the eye, the luminous efficacy of low-pressure sodium lamps is high. They are by far the most efficient light source available at present. Finally, they emit hardly any radiation in the non-visible parts of the spectrum. On these grounds, the application of low-pressure sodium lamps near sites of astronomical observatories is universally recommended. It should be noted that low-pressure sodium lamps usually prove to be a very economic proposition to begin with!

If, for specific reasons, the use of low-pressure sodium lamps is impossible or undesirable, the least one can do is to restrict the emission or radiation near the ends of the visible region of the spectrum, e.g. the far blue and the far red. Radiation emitted in these regions contributes only little to the visual sensation (more precisely to the luminance), but it may cause considerable disturbance for certain types of astronomical observations. Therefore, most bylaws and ordinances for areas near observatories restrict the emission or radiation in these spectral regions. This can be done quite simply by means of filters that can be incorporated in the lanterns.

c) Light control: We discussed the possibilities to reduce light spill by means of light control. The first measure is to avoid light being emitted above the horizon; more precisely, it means that the light is directed to the objects to be illuminated. Regarding road lighting, the measures to reduce light trespass are very similar to those that enhance the (economic) efficiency of the light installation. The reason that lanterns with optimal light control are not applied universally is twofold: primarily, lighting design schemes employing such lanterns usually require higher initial costs, whereas the economic profits are found only in the longer term - a matter that is often prohibitive in political decisions; secondly, setting up such schemes requires a high degree of light-technical skill.

d) Reduction of the amount of reflected or scattered light: Theoretically speaking, this remedy is quite important. In practice, however, the possibilities to manipulate the reflective characteristics in the real world are very limited indeed. We indicated also that reducing the reflection of the road surface is counterproductive in this respect.

The effect of air pollution might be included here. Air pollution often creates haze, or even clouds of smoke and dust; the light from the open air lighting installations often is partly reflected by these clouds, and partly absorbed. The influence of air pollution on the sky glow, particularly of faraway observatories, therefore, is not always the same. In some cases, the sky glow may increase (when the reflection predominates); in other cases the net result may be a decrease of the sky glow (when the absorption predominates) (Anon, 1985).

\section{THE PRACTICAL SOLUTION}

In preparing this paper, a simple enquiry was made to the members of IAU Commission 50. Many of the Commission members took the trouble to answer the question, for which the author thanks them most cordially. The enquiry was aimed at a brief and simple overview. As the results are quite interesting, it is recommended to repeat the enquiry on a more scientific basis. From the answers, it is clear that almost all countries have a number - and often a large number - of professional astronomical observatories, and usually a very large number of 
amateur observatories. These observatories are not restricted to the few "clear" sites, but they are located in many densely populated and highly industrialized countries as well. A second interesting feature is that many of these countries have regulations to reduce light trespass. These regulations are set up, however, exclusively on local basis. None of the answers referred to national (or nationwide) regulations or measures. It is sad to see, however, that many other countries do not have any regulations or measures at all.

Many countries cooperate in the large international observatories in Chile, Australia, Hawaii and La Palma (Canary Islands). It seems that in these sites, which are extremely important for astronomical observations, some local regulations are in force. There is reason to believe, however, that the sites are not adequately protected for future development. As a point of interest, one might quote the La Palma case - one of the very few remaining "clear" sites in the northern hemisphere - where the pressure of economic development and tourism is large. Particularly in this case, strict governmental regulations are in place. The situation is serious, particularly in view of the very expensive hardware that is installed at that site. The public opinion, however, is favorable, not only in Spain but also in the other participating countries.

The last point comes out clearly from the enquiry. On one hand, the light trespass often causes very serious problems, but on the other hand, the public opinion usually is very favorable for astronomy. The television coverage of the encounters with Halley's comet were of great help, in spite of the fact that almost no one could actually see the comet, mainly due to light trespass! And usually the governments are inclined to support astronomy.

The enquiry was too limited in scope to allow sound conclusions to be drawn. A few suggestions can be made, however. First, because the situation usually is quite unsatisfactory, there is an urgent need for regulations to reduce light trespass, not only in the few important international observatories, but in almost all countries of the world. Second, the basis for many regulations is available in the many local regulations that are in force in several countries. They need, of course, to be adapted for more general, global application. And finally, measures to restrict light trespass usually are supported by the public.

\section{RECOMMENDATIONS}

a) It is recommended to do further research regarding the generalization of "Walker's Law" and of the 1000 lumen per capita rule-of-thumb.

b) It is recommended to adapt the design methods for outdoor lighting to the specific demands of the restriction of light trespass.

c) It is recommended to promote the design and marketing of lighting equipment suitable to restrict light trespass.

d) It is recommended to establish a framework for regulations that can be applied, after adaptation to the national or local situation, to all sites of optical observatories.

e) It is recommended to establish a more systematic and more permanent form of cooperation and collaboration between the worlds of astronomers and lighting engineers. 


\section{REFERENCES}

Anon (1984). La protection des observatoires astronomiques et geophysiques. Rapport du Groupe du Travail. Institut de France, Academie des Sciences, Grasse, 1984.

Anon (1985). Identification and protection of existing and potential observing sites. (Draft). Report IAU Commission 50. International Astronomical Union, New Delhi, 1985.Anon (1985a). A statement on astronomical light pollution and light trespass. Journal of IES 14 (1985) 658-662.

CIE (1977). Measures of road lighting effectiveness. Symposium, July 5-6, 1977. CIE, Karlsruhe, 1977.

CIE (1977a). International recommendations for the lighting of roads for motorized traffic. Publication 12/2. CIE, Paris, 1977.

CIE (1980). Proceedings 19th session Kyoto 1979. Publication No. 50. CIE, Paris, 1980.

CIE (1988). Road lighting and accidents. Publication No. 8-2 (Draft, in preparation).

Crawford, D. L. (1983). Astronomy's problem with light pollution. Tucson, 1983 (year estimated).

Crawford, D. L. (1985). Light pollution: Astronomy's problem with urban sky glow. (Draft). Tucson, 1985 (year estimated).

Finch, D. M., Jewell, J. E., Leite, M. J. and Nelson, B. (1979). Atmospheric light pollution. In: CIE (1980).

Fisher, A. J. and Hall, R. R. (1977). Road lighting as an accident countermeasure. In: CIE, 1977.

IAU/CIE (1980). Guidelines for minimizing urban sky glow near astronomical observatories. Publication of IAU and CIE No. 1. CIE, Paris, 1984.

OECD (1971). Lighting, visibility and accidents. OECD, Paris, 1971.

Schreuder, D. A. (1977). The relation between lighting parameters and transportation performance. In: CIE (1977).

Schreuder, D. A. (1983). De relatie tussen verkeersongevallen en openbare verlichting (The relation between traffic accidents and public lighting). $R$ 83-12. SWOV, Leidschendam, 1983.

Schreuder, D. A. (1984). Visibility aspects of road lighting. In: TRB (1984).

Schreuder, D. A. (1987). Road lighting and light trespass. Vistas in Astronomy 30 (1987) 185-195.

TRB (1984). Providing visibility and visual guidance to the road user. Symposium July 30 - August 1, 1984. Transportation Research Board, Washington, DC, 1984.

Walker, M. F. (1973). Light pollution in California and Arizona. Publ. Astron. Soc. Pacific 85 (1973): 508-519. 